event lasted less than one minute, and $80 \%$ had prodromal symptoms. The syncope episodes occurred while standing (48\%), abrupt stand-up (19\%), seating (14\%), walking (14\%) and during or immediately after exercising (9\%).

Psychological stress (24\%), hyperventilation (10\%), trauma or blood-work (19\%) were often present. $28 \%$ had previously known headache, migraine, diabetes and rhythm disorders.

ECG was conducted in $70 \%$ (2\% pathological), EEG in $20 \%$ (1\% pathological) and blood work in 57\% (6\% pathological). Neuropediatric (13\%), cardiologic (12.9\%) and psychiatric $(6 \%)$ examinations were performed $(0,1 \%$ and $2 \%$ pathological, respectively). $22 \%$ were admitted in the hospital for further diagnostics and treatment due to diagnostic dilemma, recurrent syncope or new symptoms.

At release, $70.8 \%$ were diagnosed with reflex syncope $(63.5 \%$ vasovagal and $7.3 \%$ situational), hyperventilation (7.3\%), heat illness (4.1\%), pseudo syncope (4.2\%), breath holding spells (4.2\%), orthostatic syncope (3.1\%), convulsions (3.2\%), toxin exposure $(2.1 \%)$ and anaphylaxis $(1 \%)$.

The majority of the children experiencing syncope in pediatric ED were not life endangered by cardiac or other underlying etiology. A potentially life-threatening was an anaphylactic shock, toxin exposed children, heat syncope and exercise associated collapse. Based on a detailed history, clinical exam and specific diagnostic procedures, specialists in ED should recognize specific conditions that require attention.

\section{COMBINED INTRANASAL DEXMEDETOMIDINE AND KETAMINE VS INTRANASAL DEXMEDETOMIDINE AND ORAL MIDAZOLAM FOR PROCEDURAL SEDATION IN CHILDREN: A RANDOMIZED MULTICENTRE TRIAL TO EVALUATE INDUCTION TIME AND SEDATION LEVEL}

Andrea Trombetta*, Francesca Cossovel, Egidio Barbi. University of Trieste

10.1136/archdischild-2021-europaediatrics.335

The aim of this study is to compare the combination of intranasal dexmedetomidine and ketamine to intranasal dexmedetomidine and oral midazolam to evaluate induction time and sedation level in children undergoing procedural sedation.

this multicentre trial was conducted in a tertiary pediatric teaching hospital and in a secondary hospital on patients in need of procedural sedation who referred from November 2019 to March 2020. All consecutively admitted subjects were randomized to receive one-time dose $(4 \mathrm{mcg} / \mathrm{kg}$ ) of intranasal dexmedetomidine and $3 \mathrm{mg} / \mathrm{Kg}$ of intranasal ketamine or onetime dose of $4 \mathrm{mcg} / \mathrm{Kg}$ of intranasal dexmedetomidine and oral midazolam $(0.5 \mathrm{mg} / \mathrm{Kg})$ with the intended goal to compare induction-time, effectiveness and adverse effects.

fifty patients were recruited to receive intranasal dexmedetomidine and ketamine and fifty patients were recruited to receive intranasal dexemdetomidine and oral midazolam. The induction time was significantly lower in the intervention group when compared to the control group (11' and 33 minutes, respectively). Sedation success rate was similar in both groups without any major adverse observed in both groups according to this preliminary report a combination of intranasal dexmedetomidine and ketamine could be considered as a possible option in for procedural sedation in children, in particular in settings in which shortening induction-time is essential in comparison to an oral midazolam plus in dexmedetomidine association.

\section{THROMBOEMBOLIC EVENT AND IT'S MANAGEMENT IN A GIRL WITH RESTRICTIVE CARDIOMYOPATHY AND IMPLANTED BERLIN HEART EXCOR DEVICE}

Mario Šestan*, Hana Matković, Sandro Dessardo, Toni Matić, Miran Cvitković, Slobodan Galić, Dorotea Bartoniček, Marko Radoš, David Ozretić, Ivan Jovanović, Filip Rubić. Department of Paediatrics, University Hospital Centre Zagreb; School of medicine, University of Zagreb

\subsection{6/archdischild-2021-europaediatrics.336}

Ventricular assist devices are increasingly used as an emerging therapy in children with different heart condition, mostly cardiomyopathy. Introduction of pulsatile devices for bridge to heart transplantation contributed to significant improvements in the survival and discharge rate of these patients. Berlin Heart EXCOR Pediatric (BH) is a pulsatile device with different chamber sizes and is registered for use in pediatric patients. When such devices are used during longer period of time, many complications can occur, most of which are linked to clotting disorders. Therefore, Edmonton protocol was introduced for patients on $\mathrm{BH}$ where detailed instructions are given regarding institution and maintenance of anticoagulation and antiaggregation therapy. Despite proper management, patients could have devastating complications, particularly cerebral arterial thromboses.

We present a case of a 2,5-year-old girl who was diagnosed with restrictive cardiomyopathy caused by the p.Arg192Cys variant in the gene encoding cardiac troponin I (TNNI3). When her condition worsened and end-stage heart failure developed, she was placed on Venoarterial extracorporeal membrane oxygenation (VA-ECMO), by which she was supported for 3 weeks. Since she did not tolerate separation from mechanical support, a left ventricular assist device $\mathrm{BH}$ was implanted. Afterwards her anticoagulation and antiaggregation therapy was managed according Edmonton protocol thus she was given warfarin (target INR 2.8-3.5), acetylsalicylic acid (target value of ASPI test $<30 \mathrm{U}$ ) and clopidogrel (target value of ADP test $<50 \mathrm{U}$ ). After 50 days on $\mathrm{BH}$ significant changes in behavior with a qualitative change in the level of consciousness were observed in our patient prompting a computed tomographic cerebral angiography to be performed, resulting in the detection of a thrombus in the right middle cerebral artery. Motor deficit i.e. paresis of her left limbs was not present during the first hours of symptom development and appeared soon after brain imaging was done. After urgent consultation with our interventional radiologists, she underwent thrombectomy through her right femoral artery with complete reperfusion of the affected parts of the brain followed by immediate recovery of her mental state while her motor deficit resolved over the next 2 weeks.

Clinicians should have a low threshold for early imaging of central nervous system when arterial thromboses are suspected. Good cooperation with radiologists that can perform such minimally invasive procedures is of the most importance since it can be lifesaving. 